\title{
Brief daily postpartum separations from the litter alter dam response to psychostimulants and to stress
}

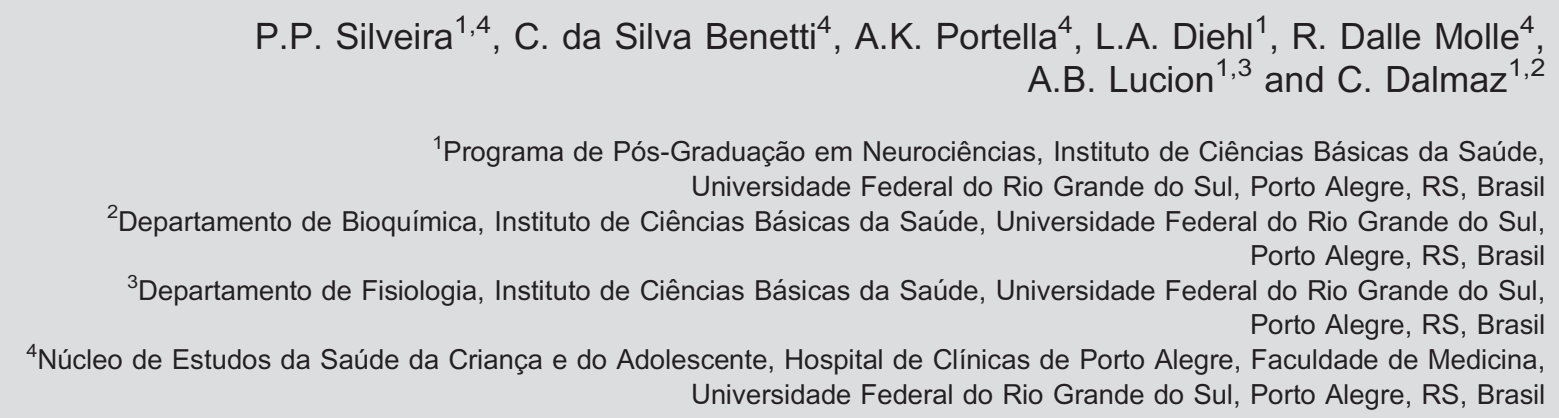

\begin{abstract}
Neonatal handling induces several behavioral and neurochemical alterations in pups, including decreased responses to stress and reduced fear in new environments. However, there are few reports in the literature concerning the behavioral effects of this neonatal intervention on the dams during the postpartum period. Therefore, the aim of the current study was to determine if brief postpartum separation from pups has a persistent impact on the dam's stress response and behavior. Litters were divided into two neonatal groups: 1) non-handled and 2) handled [10 min/day, from postnatal day (PND) 1 to 10]. Weaning occurred at PND 21 when behavioral tasks started to be applied to the dams, including sweet food ingestion (PND 21), forced swimming test (PND 28), and locomotor response to a psychostimulant (PND 28). On postpartum day 40, plasma was collected at baseline for leptin assays and after $1 \mathrm{~h}$ of restraint for corticosterone assay. Regarding sweet food consumption, behavior during the forced swimming test or plasma leptin levels did not differ between dams briefly separated and non-separated from their pups during the postpartum period. On the other hand, both increased locomotion in response to diethylpropion and increased corticosterone secretion in response to acute stress were detected in dams briefly separated from their pups during the first 10 postnatal days. Taken together, these findings suggest that brief, repeated separations from the pups during the neonatal period persistently impact the behavior and induce signs of dopaminergic sensitization in the dam.
\end{abstract}

Key words: Feeding behavior; Neonatal handling; Psychostimulants; Stress response; Dam behavior

\section{Introduction}

It is widely known that disturbing the mother-pup interaction can produce long-lasting effects in the offspring $(1,2)$. After just a brief period of separation from the mother (neonatal handling) animals may show more efficient resolution of the hormonal discharge after acute stress (3); early handling has also been shown to increase the ability to cope with anxiety and fear in adulthood (4). Handled animals have been reported to show a series of stable neurochemical modifications that influence their behavior and susceptibility to different conditions (5-8).

These findings can be associated with changes in maternal behavior caused by the temporary interruption in the dam-pup relationship. Mothers of handled litters become more intense caretakers when the pups are returned to the litter; these mothers groom and lick their pups at significantly increased frequency $(9,10)$. It has been reported that the bouts of maternal licking and grooming behavior are accompanied by an increase in nucleus accumbens dopamine release in the dam (11). It is suggested that the neurotransmitter plays an important role in the mediation of the maternal expression of caring for the pup.

The literature shows evidence that mothers bar-press for their pups (12); it also shows that dams may develop 
conditioned place preference for a pup cue-associated chamber versus a cocaine cue-associated chamber (13). The behavioral paradigms developed in these studies require specific actions of dopamine neurotransmission (14). Minimal blockade of dopamine interferes with maternal motivation of lactating rats to maintain contact with pups (15). Natural variations in maternal care are associated with variations in dopamine signaling in the nucleus accumbens during bouts of licking and grooming; these variations may be regulated by oxytocin at the level of the ventral tegmental area (16).

It is interesting to note that leptin, a hormone that exerts a variety of regulatory functions especially related to feeding regulation and energy balance, has been recently described to modulate the mesolimbic dopamine system (17) and hypothalamus-pituitary-adrenal (HPA) axis response to stress (18) as well. Additionally, leptin has been proposed to regulate dopaminergic neurotransmission in response to stressor stimuli in humans (19).

Because neonatal handling profoundly affects maternal care $(9,10)$, and this behavior is deeply influenced by the dopamine signaling in mesolimbic regions as reviewed above, we hypothesized that repeated brief separation from the pups could act as a repeated mild stressor for the mother, therefore sensitizing her dopaminergic mesolimbic system, possibly persistently, with an impact on behaviors such as palatable food intake and vulnerability to depression. To our knowledge, few studies have investigated the long-term effects of brief neonatal separations from the litter on the dams $(20,21)$. Our objective was to determine whether brief, repeated separation from the pups during the neonatal period would persistently impact the behavior of the dams, and whether it would induce signs of dopaminergic sensitization in the dam.

\section{Material and Methods}

\section{Animals}

Pregnant Wistar rats were randomly selected from our animal facility. They were housed individually in $65 \times 25$ $\times 15-\mathrm{cm}$ home cages made of Plexiglas with sawdustcovered floors. The rats were kept in a controlled environment until parturition: lights on between 7:00 am and 7:00 pm and temperature of $22 \pm 2{ }^{\circ} \mathrm{C}$. Pups were culled to 8 per litter within $24 \mathrm{~h}$ after birth and were maintained non-handled except for the separation procedure. The procedure was carried out between 10:00 am and 3:00 pm. All animal procedures were approved by the Institutional Ethics Committee of Universidade Federal do Rio Grande do Sul (UFRGS) and GPPG/HCPA (FIPE \#10-0525).

\section{Daily postpartum separations from the litter}

In the non-handled group pups were undisturbed with the dam until weaning. In the handled group, pups were removed from their home cage and placed in a clean cage lined with clean paper towels. The cage was placed inside an incubator set at $32^{\circ} \mathrm{C}$. After $10 \mathrm{~min}$, the pups were returned to their dams. The procedure was performed during the first 10 days of life. After 10 days, the litter was left undisturbed until postpartum day 21 , at which time the pups were weaned. At weaning, dams were removed gently from their litter and then placed in another home cage. We placed 4 to 5 dams per cage, separated according to group (non-handled or handled litters). Fortyeight adult female rats (weight: $230-300 \mathrm{~g}$ ) were used for the study. Rats had free access to food (standard lab rat chow) and water except during the application of the behavioral tasks. The tasks were performed between 1:00 and 4:00 pm. The behavioral tasks were carried out only after weaning and following the scheme illustrated in Figure 1. Another set of dams was submitted to a different set of experiments (Figure 2).

Yet another group of dams was treated in the same manner as the groups described above, but only until weaning. After weaning, they were left undisturbed until postpartum day 40, at which time half were fasted for $6 \mathrm{~h}$ and directly exposed to single-acute restraint stress for $1 \mathrm{~h}$. Restraint stress was carried out by placing the animal in a $25 \times 7-\mathrm{cm}$ plastic tube and adjusting the tube with plaster tape on the outside so that the animal was unable to move. There was a $1-\mathrm{cm}$ hole at the far end for breathing. After exposure to acute stress, dams were decapitated and blood was collected for assay of corticosterone. The remaining dams were not submitted to restraint stress and were used as controls.

\section{Habituation and test of sweet food ingestion}

Habituation to sweet food was initiated immediately

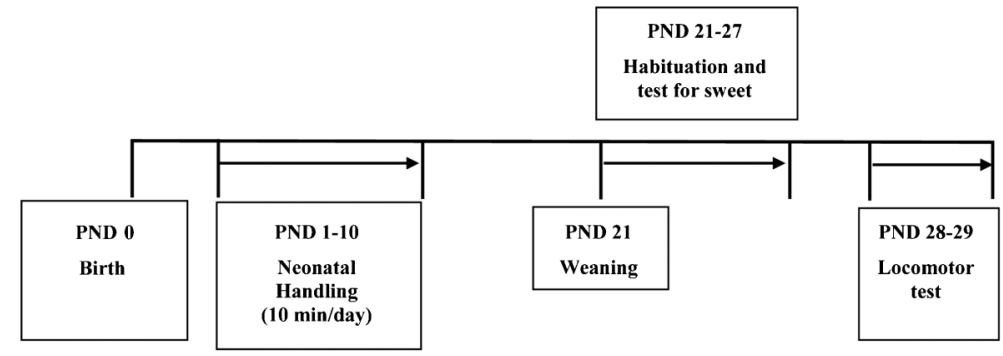

Figure 1. Scheme for the first set (locomotor test) of behavioral tasks in separated and non-separated dams on postnatal days 28 to 29 , for $\mathrm{n}=8-9$ dams/group. 


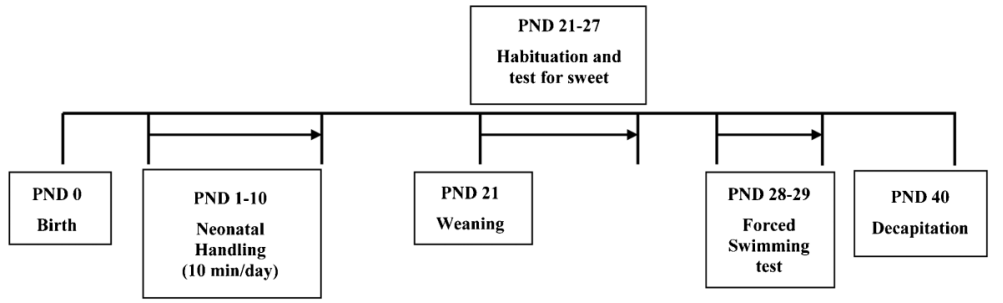

Figure 2. Scheme for the second set (forced swimming test) of behavioral tasks in separated and nonseparated dams on postnatal days 28 to 29 , for $n=9$ dams/group. after weaning on postnatal day 21. Dams were individually placed in a lit rectangular $(40 \times 15 \times 20 \mathrm{~cm})$ box; the floor and sidewalls of the box were made of wood and the box was covered with a glass ceiling. Ten Froot Loops pellets of wheat and cornstarch and sucrose (Kellogg's ${ }^{\mathbb{R}}$, Brazil) were placed on one of the extremities of the box and the dams were allowed to eat them. During the 5 days of habituation, the animals were exposed to the sweet pellets for $3 \mathrm{~min}$. On the 6 th day (test session), the dams were again exposed to the sweet pellets for $3 \mathrm{~min}$ and the number of ingested pellets was counted. A protocol was established to calculate partial eating of the Froot Loops pellets (for example, $1 / 3$ or $1 / 4$ of a pellet); the measured fractions were counted as well.

\section{Locomotor response to diethylpropion}

We administered a dose of diethylpropion, an amphetamine-type stimulant, to the rats. The goal was to examine behavioral and dopaminergic sensitization to the drug in the dams that were briefly separated from their pups during the neonatal period.

On the first day, rats were injected intraperitoneally (ip) with $1 \mathrm{mg} / \mathrm{kg}$ saline. Immediately after the injection they were kept in a neutral box for $10 \mathrm{~min}$. Next, rats were placed individually in a 50-cm high, $40 \times 60-\mathrm{cm}$ open wooden arena with a frontal glass wall. The floor was equally divided with white lines into 12 rectangles measuring $13.3 \times 15.0 \mathrm{~cm}$. The animals were observed continuously for $30 \mathrm{~min}$ by observers blind to the experimental condition. The number of crossings and rearings was registered. On the second day, rats were injected ip once with $10 \mathrm{mg} / \mathrm{kg}$ diethylpropion and the same parameters were analyzed.

\section{Forced swimming test}

Maternal separation from the pups in the immediate postpartum period can impact maternal behavior. Thus, we carried out the forced swimming test to investigate if the dams developed depression-like behavior. Animals were placed for $15 \mathrm{~min}$ in a rigid plastic cylinder measuring $33 \mathrm{~cm}$ in height and $20 \mathrm{~cm}$ in diameter. The cylinder contained tap water at $24^{\circ} \mathrm{C}$ (training session). Twenty-four hours later, animals were exposed to the same conditions and total immobility time duration was registered (5-min period, test session). A rat was judged to be immobile when it made only the movements necessary to keep its head above water level.

\section{Plasma collection and biochemical assays}

Animals were decapitated on postpartum day 40 after a 6-h fast. Trunk blood was collected into heparinized tubes for the determination of leptin and corticosterone levels. Tubes were centrifuged at $4{ }^{\circ} \mathrm{C}$ and plasma was separated and frozen until the day of the analysis. Hormone levels were measured using commercially available ELISA kits; $50 \mu \mathrm{L}$ plasma was tested for corticosterone (Cayman Chemical Co., USA; intra-assay coefficient of variation: $7.5 \%$; interassay coefficient of variation: $14.6 \%$ ) and $10 \mu \mathrm{L}$ plasma for leptin (Linco Research Inc., USA; intra-assay coefficient of variation: $2.49 \%$; interassay coefficient of variation: $3.93 \%$ ).

\section{Statistical analysis}

Data are reported as means \pm SE and were analyzed by the Student $t$-test (sweet food ingestion test, forced swimming task, and leptin parameters), two-way ANOVA (for corticosterone analysis), and repeated measures ANOVA (for sweet food habituation sessions and locomotor response); results were considered to be significant at $\mathrm{P}<0.05$.

\section{Results}

\section{Sweet food ingestion}

During the sweet food habituation, there was an effect of time, meaning that both briefly separated and nonseparated dams ( $n=12-13 /$ group) ate significantly more sweet food as the days progressed $[F(1,23)=17.023, P$ $<0.0001]$; but there was no group effect alone $[\mathrm{F}(1,23)=$ $1.969, P=0.174]$ (see Figure 3 ). In the test session,

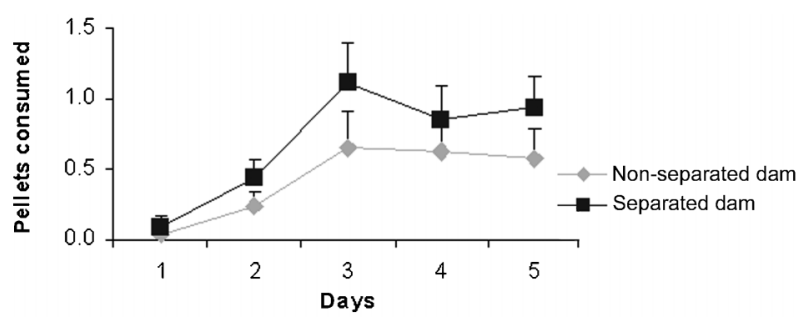

Figure 3. Consumption of sweet pellets during habituation. Data are reported as the mean \pm SE number of pellets eaten. Both groups of dams (separated and non-separated) increased sweet food consumption as the days progressed (effect of time, $\mathrm{P}<$ 0.0001 , repeated measures ANOVA, $n=12-13$ dams/group). 
there was no significant difference on any of the days between the groups of dams in terms of sweet food consumption (non-separated: $0.86 \pm 0.27$ vs separated: $0.87 \pm 0.29$, Student $t$-test, $\mathrm{t}(23)=0.016, \mathrm{P}=0.988)$.

\section{Locomotor response to diethylpropion}

During the locomotion task, diethylpropion was associated with a significant increase in crossings $[F(1,15)=$ 234.436, $\mathrm{P}<0.0001$; repeated measures ANOVA], in both briefly separated and non-separated dams ( $n=8-9 /$ group). A significant interaction was found between diethylpropion and neonatal handling $[F(1,15)=8.801, P=0.01]$ and $a$ separate effect of handling $[F(1,15)=5.509, P=0.032]$ was identified. The effect showed that dams briefly separated from their litters during the postpartum period responded to diethylpropion with more locomotion compared to non-separated dams. The number of rearings was also increased by the drug $[F(1,15)=23.203, P=0.001$; repeated measures ANOVA], but there was no interaction between drug and group $[F(1,15)=0.925, P=0.351]$ (see Figure 4A and B).

\section{Forced swimming test}

Total immobility time (in seconds) in the forced swimming test was not significantly different between
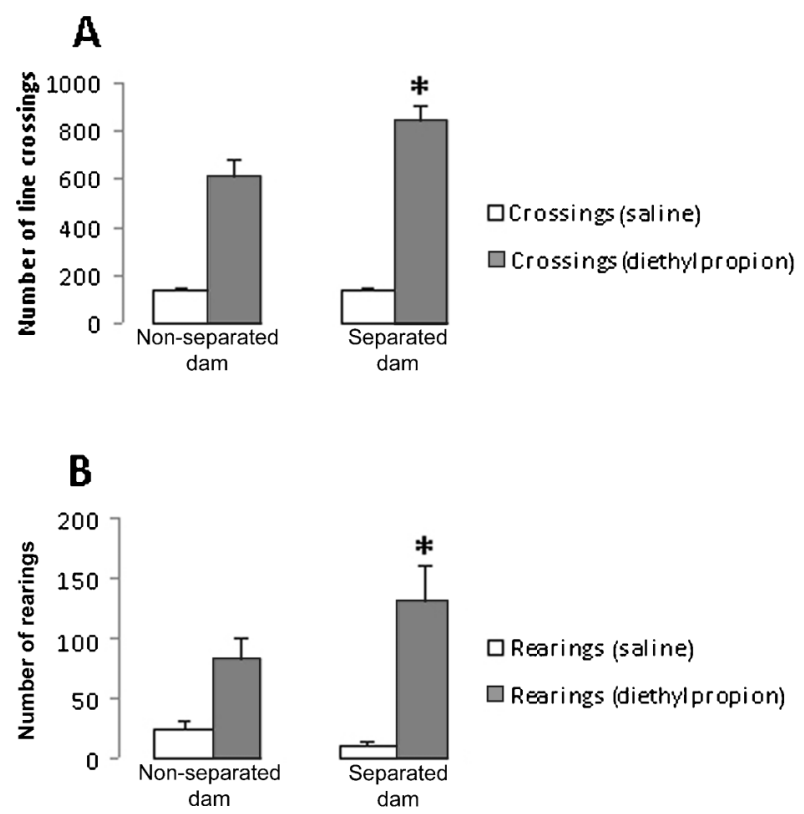

Figure 4. Behavioral profile in response to an intraperitoneal psychostimulant injection (10 mg/kg diethylpropion). Data are reported as means $\pm \mathrm{SE}$. $A$, Number of crossings was increased by the drug in comparison with saline $(P<0.0001$, repeated measures ANOVA, $\mathrm{n}=8-9$ dams/group), especially in separated dams ( ${ }^{*} P=0.032$ for the group $x$ drug interaction). $B$, Number of rearings was also increased by the drug compared to saline $(P=$ $0.001)$ without interaction between drug and group $(P>0.05)$. briefly separated and non-separated dams ( = 9/group; non-separated: $242.66 \pm 25.74$ vs separated: $220.44 \pm$ 26.39, $\mathrm{t}(17)=0.363, \mathrm{P}=0.555$, ).

\section{Plasma studies}

Plasma leptin levels were not significantly different between dams ( $\mathrm{n}=7 /$ group) briefly separated or not separated from their litters $[\mathrm{t}(12)=1.239, \mathrm{P}=0.239$; Student $t$-test]. As expected, plasma corticosterone was affected by exposure to stress $[\mathrm{F}(1,26)=9.565, \mathrm{P}=$ 0.005 ; two-way ANOVA], with dams ( $\mathrm{n}=6-7 /$ group) exposed to restraint stress exhibiting increased corticosterone levels compared to control dams. There was an interaction between acute stress exposure and dam group $[F(1,26)=4.367, \mathrm{P}=0.048]$; in other words, separated dams had higher corticosterone secretion in response to acute stress than non-separated dams (Table 1).

\section{Discussion}

The present findings indicate that brief and repeated separation from the pups during the neonatal period can change the HPA stress axis and locomotor response to psychostimulants in dams for as long as 40 days postpartum. The dams showed enhanced behavioral sensitivity to diethylpropion (an amphetamine analogue) associated with increased locomotor activity. We also identified an increased corticosterone release in response to restraint stress; for the dams, this represented an unknown or new stressor. The results are consistent with our hypothesis that brief, repeated separations from the pups during the neonatal period have a persistent impact on maternal behavior and induce signs of dopaminergic sensitization in the dams. Few studies have investigated this issue. In one of them, Kalinichev et al. (20) reported that periodic postpartum separation from the pups induces behavioral sensitization to morphine in the dams (20).

Evidence has shown that repeated exposure to stress results in an increased locomotor response to a psychostimulant challenge (22). In our study, it is possible that brief and repeated separation from the pups acted as a repeated stressor, and that this led to cross-sensitization expressed as an increased locomotor response to diethylpropion.

Other studies have reported that exposure to stressors causes sensitization of the HPA axis response to heterotypic stressors $(23,24)$. Similarly, the present study suggests that the neonatal handling procedure applied sensitizes dam HPA activity in response to a heterotypic stressor (restraint). Alternatively, the mesolimbic dopamine system may be implicated in the behavioral sensitization described in our study $(25,26)$. The intervention in the mother-pup dyad during the postpartum period is possibly associated with repeated peaks of accumbal dopamine release; these peaks, in turn, are associated with the bursts of licking and grooming that 
Table 1. Plasma leptin levels at baseline and corticosterone levels at baseline and after 1-h restraint stress.

\begin{tabular}{|c|c|c|c|}
\hline \multirow[t]{2}{*}{ Group } & \multirow[t]{2}{*}{ Plasma leptin (ng/mL) } & \multicolumn{2}{|c|}{ Plasma corticosterone (ng/mL) } \\
\hline & & Baseline & After 1-h restraint stress \\
\hline Non-separated & $0.85 \pm 0.12$ & $192.54 \pm 58.84$ & $225.04 \pm 64.33^{\#}$ \\
\hline Separated & $1.03 \pm 0.08$ & $156.93 \pm 64.61$ & $324.87 \pm 128.66^{\# *}$ \\
\hline
\end{tabular}

Leptin levels did not differ between separated and non-separated dams $(\mathrm{P}>0.05$, Student $t$-test, $\mathrm{n}=7$ dams/group). Plasma corticosterone levels were increased by exposure to stress in both groups ( ${ }^{\#} P=0.005$, two-way ANOVA, $n=6-7$ dams/group), markedly in separated dams compared to non-separated dams ( ${ }^{*} \mathrm{P}=0.048$ for the interaction).

occur upon reunion with the pup (11).

Similarly to stress, glucocorticoids facilitate the psychomotor-stimulant effects of cocaine (27) and amphetamine (28). Glucocorticoids have facilitatory effects on dopaminergic activity. For instance, suppression of glucocorticoids by adrenalectomy decreases the induction of Fos proteins in response to an ip injection of the dopamine D1 agonist (29). Glucocorticoids have also been shown to increase dopamine synthesis by acting on tyrosine hydroxylase activity (30), to decrease monoamine-oxidase activity (31) and to decrease dopamine reuptake (32).

It has been shown that viral-mediated increase in the expression of 5-hydroxytryptamine 1B receptor (5-HT 1B) receptors on the terminals of the nucleus accumbens shell followed by repeated exposure to mild stress subsequently enhances the acute locomotor-activating effects of amphetamine in rats (33). This result suggests that accumbal 5-HT $1 \mathrm{~B}$ receptors may also be involved in our findings.

We also showed that dams whose offspring were subjected to repeated handling showed no significant difference in immobility time in the forced swimming test when compared to controls. This result agrees with the findings of other investigators who observed that only dams subjected to longer separations from their pups show increased immobility time $(34,35)$; the latter result is consistent with depression-like behavior (36). Although high levels of corticosterone or stress in the postpartum period impair maternal care $(37,38)$, others have reported that mild increases in maternal corticosterone levels (similar to those induced by exposure to a mild stressor) actually increase maternal care (39), being protective against brain ischemic insults in the adult offspring. This is possibly an adaptive response in which subtle environ-

\section{References}

1. Levine S. Infantile experience and resistance to physiological stress. Science 1957; 126: 405, doi: 10.1126/science. 126.3270.405.

2. Meaney MJ, Diorio J, Francis D, Widdowson J, LaPlante P, Caldji C, et al. Early environmental regulation of forebrain glucocorticoid receptor gene expression: implications for mental stress increases maternal care to guarantee offspring survival.

Leptin is a proinflammatory cytokine associated with regulatory functions, especially those related to food intake and energy homeostasis. It has also been shown to influence the mesolimbic dopaminergic system (17). In the present study, we did not find significant differences in leptin levels between the groups of dams tested. This result suggests that these hormone levels are not related to the dopaminergic sensitization reported. Some investigators have described interactions of leptin in controlling the HPA axis activity and modulating stress adaptation via the same axis (18). We cannot exclude the possibility that the absence of difference in leptin levels between briefly separated and control dams is associated with differential leptin sensitivity in the dams, since stress (brief separation is described as a slight stressor event) up-regulates peripheral leptin receptors (40).

Our results suggest that brief separation from the pup during the postpartum period induces behavioral sensitization to psychostimulants in dams. It also induces increased corticosterone secretion in response to acute stress in the dams. As these alterations are observed 40 days after parturition, they may be considered to be longlasting changes induced by the neonatal handling procedure, and may be involved in the persistent changes in maternal care induced by this intervention (5). These findings may contribute to the understanding of meaningful relationships between mother and offspring in early life.

\section{Acknowledgments}

Research supported by CNPq, CAPES, and FIPE (\#10-0525).

adrenocortical responses to stress. Dev Neurosci 1996; 18 49-72, doi: 10.1159/000111395.

3. Panagiotaropoulos T, Papaioannou A, Pondiki S, Prokopiou A, Stylianopoulou F, Gerozissis K. Effect of neonatal handling and sex on basal and chronic stress-induced corticosterone and leptin secretion. Neuroendocrinology 
2004; 79: 109-118, doi: 10.1159/000076633.

4. Núñes JF, Ferré P, García E, Escorihuela RM, FernandésTeruel A, Tobeña A. Postnatal handling reduces emotionality ratings and accelerates two-way active avoidance in female rats. Physiol Behav 1994; 57: 831-835, doi: 10.1016/ 0031-9384(94)00308-R.

5. Liu D, Diorio J, Tannenbaum B, Caldji C, Francis D, Freedman A, et al. Maternal care, hippocampal glucocorticoid receptors, and hypothalamic-pituitary-adrenal responses to stress. Science 1997; 277: 1659-1662, doi: 10.1126/science.277.5332.1659.

6. Portella AK, Silveira PP, Diehl LA, Crema LM, Clemente Z, Peres $W$, et al. Early life handling decreases serotonin turnover in the nucleus accumbens and affects feeding behavior of adult rats. Dev Psychobiol 2010; 52: 190-196.

7. Silveira PP, Portella AK, Benetti CS, Zugno Al, Scherer EB, Mattos $\mathrm{CB}$, et al. Association between $\mathrm{Na}(+), \mathrm{K}(+)$-ATPase activity and the vulnerability/resilience to mood disorders induced by early life experience. Neurochem Res 2011; 36: 2075-2082, doi: 10.1007/s11064-011-0531-1.

8. Silveira PP, da Silva BC, Ayres C, Pederiva FQ, Portella $A K$, Lucion $A B$, et al. Satiety assessment in neonatally handled rats. Behav Brain Res 2006; 173: 205-210, doi: 10.1016/j.bbr.2006.06.031.

9. Cirulli F, Berry A, Alleva E. Early disruption of the motherinfant relationship: effects on brain plasticity and implications for psychopathology. Neurosci Biobehav Rev 2003; 27: 73-82, doi: 10.1016/S0149-7634(03)00010-1.

10. Branchi I, Santucci D, Alleva E. Ultrasonic vocalisation emitted by infant rodents: a tool for assessment of neurobehavioural development. Behav Brain Res 2001; 125: 49-56, doi: 10.1016/S0166-4328(01)00277-7.

11. Champagne FA, Chretien $P$, Stevenson CW, Zhang TY, Gratton A, Meaney MJ. Variations in nucleus accumbens dopamine associated with individual differences in maternal behavior in the rat. $J$ Neurosci 2004; 24: 4113-4123, doi: 10.1523/JNEUROSCI.5322-03.2004.

12. Lee A, Clancy S, Fleming AS. Mother rats bar-press for pups: effects of lesions of the mpoa and limbic sites on maternal behavior and operant responding for pup-reinforcement. Behav Brain Res 2000; 108: 215-231, doi: 10.1016/ S0166-4328(99)00170-9.

13. Mattson BJ, Williams S, Rosenblatt JS, Morrell JI. Comparison of two positive reinforcing stimuli: pups and cocaine throughout the postpartum period. Behav Neurosci 2001; 115: 683-694, doi: 10.1037/0735-7044.115.3.683.

14. Doyon WM, Ramachandra V, Samson HH, Czachowski CL, Gonzales RA. Accumbal dopamine concentration during operant self-administration of a sucrose or a novel sucrose with ethanol solution. Alcohol 2004; 34: 261-271, doi: 10.1016/j.alcohol.2004.10.004.

15. Stern JM, Keer SE. Maternal motivation of lactating rats is disrupted by low dosages of haloperidol. Behav Brain Res 1999; 99: 231-239, doi: 10.1016/S0166-4328(98)00108-9.

16. Shahrokh DK, Zhang TY, Diorio J, Gratton A, Meaney MJ. Oxytocin-dopamine interactions mediate variations in maternal behavior in the rat. Endocrinology 2010; 151: 2276-2286, doi: 10.1210/en.2009-1271.

17. Opland DM, Leinninger GM, Myers MG Jr. Modulation of the mesolimbic dopamine system by leptin. Brain Res 2010; 1350: 65-70, doi: 10.1016/j.brainres.2010.04.028.
18. Roubos EW, Dahmen M, Kozicz T, Xu L. Leptin and the hypothalamo-pituitary-adrenal stress axis. Gen Comp Endocrino/2012; 177:28-36, doi: 10.1016/j.ygcen.2012.01.009.

19. Burghardt PR, Love TM, Stohler CS, Hodgkinson C, Shen $\mathrm{PH}$, Enoch MA, et al. Leptin regulates dopamine responses to sustained stress in humans. J Neurosci 2012; 32: 1536915376, doi: 10.1523/JNEUROSCI.2521-12.2012.

20. Kalinichev M, Easterling KW, Holtzman SG. Long-lasting changes in morphine-induced locomotor sensitization and tolerance in Long-Evans mother rats as a result of periodic postpartum separation from the litter: a novel model of increased vulnerability to drug abuse? Neuropsychopharmacology 2003; 28: 317-328, doi: 10.1038/sj.npp.1300068.

21. Boccia ML, Pedersen CA. Brief vs. long maternal separations in infancy: contrasting relationships with adult maternal behavior and lactation levels of aggression and anxiety. Psychoneuroendocrinology 2001; 26: 657-672, doi: 10.1016/S0306-4530(01)00019-1.

22. Covington HE III, Miczek KA. Repeated social-defeat stress, cocaine or morphine. Effects on behavioral sensitization and intravenous cocaine self-administration "binges". Psychopharmacology 2001; 158: 388-398, doi: 10.1007/ s002130100858.

23. Belda X, Fuentes S, Nadal R, Armario A. A single exposure to immobilization causes long-lasting pituitary-adrenal and behavioral sensitization to mild stressors. Horm Behav 2008; 54: 654-661, doi: 10.1016/j.yhbeh.2008.07.003.

24. Hauger RL, Lorang M, Irwin M, Aguilera G. CRF receptor regulation and sensitization of $\mathrm{ACTH}$ responses to acute ether stress during chronic intermittent immobilization stress. Brain Res 1990; 532: 34-40, doi: 10.1016/0006-8993(90)91738-3.

25. Clarke PB, Fu DS, Jakubovic A, Fibiger HC. Evidence that mesolimbic dopaminergic activation underlies the locomotor stimulant action of nicotine in rats. $J$ Pharmacol Exp Ther 1988; 246: 701-708.

26. Robinson TE, Berridge KC. Incentive-sensitization and addiction. Addiction 2001; 96: 103-114, doi: 10.1046/ j.1360-0443.2001.9611038.x.

27. Marinelli M, Piazza PV, Deroche V, Maccari S, Le Moal M, Simon $\mathrm{H}$. Corticosterone circadian secretion differentially facilitates dopamine-mediated psychomotor effect of cocaine and morphine. J Neurosci 1994; 14: 2724-2731.

28. Cador M, Cole BJ, Koob GF, Stinus L, Le Moal M. Central administration of corticotropin releasing factor induces longterm sensitization to D-amphetamine. Brain Res 1993; 606: 181-186, doi: 10.1016/0006-8993(93)90982-S.

29. Barrot M, Abrous DN, Marinelli M, Rouge-Pont F, Le Moal M, Piazza PV. Influence of glucocorticoids on dopaminergic transmission in the rat dorsolateral striatum. Eur J Neurosci 2001; 13: 812-818, doi: 10.1046/j.1460-9568.2001.01434.x.

30. Ortiz J, DeCaprio JL, Kosten TA, Nestler EJ. Strainselective effects of corticosterone on locomotor sensitization to cocaine and on levels of tyrosine hydroxylase and glucocorticoid receptor in the ventral tegmental area. Neuroscience 1995; 67: 383-397, doi: 10.1016/03064522(95)00018-E.

31. Caesar PM, Collins GG, Sandler M. Catecholamine metabolism and monoamine oxidase activity in adrenalectomized rats. Biochem Pharmacol 1970; 19: 921-926, doi: 10.1016/0006-2952(70)90255-8.

32. Gilad GM, Rabey JM, Gilad VH. Presynaptic effects of 
glucocorticoids on dopaminergic and cholinergic synaptosomes. Implications for rapid endocrine-neural interactions in stress. Life Sci 1987; 40: 2401-2408, doi: 10.1016/00243205(87)90754-5.

33. Ferguson SM, Sandygren NA, Neumaier JF. Pairing mild stress with increased serotonin-1B receptor expression in the nucleus accumbens increases susceptibility to amphetamine. Eur J Neurosci 2009; 30: 1576-1584, doi: 10.1111/ j.1460-9568.2009.06933.x.

34. Boccia ML, Razzoli M, Vadlamudi SP, Trumbull W, Caleffie C, Pedersen CA. Repeated long separations from pups produce depression-like behavior in rat mothers. Psychoneuroendocrinology 2007; 32: 65-71, doi: 10.1016/ j.psyneuen.2006.10.004.

35. Maniam J, Morris MJ. Long-term postpartum anxiety and depression-like behavior in mother rats subjected to maternal separation are ameliorated by palatable high fat diet. Behav Brain Res 2010; 208: 72-79, doi: 10.1016/ j.bbr.2009.11.005.

36. von Pose Toigo E, Diehl LA, Ferreira AG, Mackedanz V, Krolow R, Benitz AN, et al. Maternal depression model: long-lasting effects on the mother following separation from pups. Neurochem Res 2012; 37: 126-133, doi: 10.1007/ s11064-011-0590-3.

37. Nephew BC, Bridges RS. Effects of chronic social stress during lactation on maternal behavior and growth in rats. Stress 2011; 14: 677-684.

38. Workman JL, Brummelte S, Galea LA. Postpartum corticosterone administration reduces dendritic complexity and increases the density of mushroom spines of hippocampal CA3 arbours in dams. J Neuroendocrinol 2013; 25: 119130, doi: 10.1111/j.1365-2826.2012.02380.x.

39. Casolini P, Domenici MR, Cinque C, Alema GS, Chiodi V, Galluzzo M, et al. Maternal exposure to low levels of corticosterone during lactation protects the adult offspring against ischemic brain damage. J Neurosci 2007; 27: 70417046, doi: 10.1523/JNEUROSCI.1074-07.2007.

40. Morera P, Basirico L, Hosoda K, Bernabucci U. Chronic heat stress up-regulates leptin and adiponectin secretion and expression and improves leptin, adiponectin and insulin sensitivity in mice. J Mol Endocrinol 2012; 48: 129-138, doi: 10.1530/JME-11-0054. 